[RADIOCARBon, V́ol. 20, No. 3, 1978, P. 469-486]

\title{
UPPSALA NATURAL RADIOCARBON MEASUREMENTS XII
}

INGRID U OLSSON and M FARID A F EL-DAOUSHY

Fysiska institutionen, Uppsala universitet, Uppsala, Sweden

The following list covers all bone sample measurements finished since the last list (R, 1972, v 14, p 247-271) until the end of 1977; the Egyptian samples were measured as an interlaboratory project and all shells were closely related to some of the bone samples.

The technique and the pretreatment are mainly the same as previously described (Olsson, 1958). In autumn 1970, we changed from bottles filled with saturated $\mathrm{CrO}_{3}$ in conc $\mathrm{H}_{2} \mathrm{SO}_{4}$ to another solution (a mixture of $60 \mathrm{~g} \mathrm{CrO}_{3}, 60 \mathrm{ml} \mathrm{H}_{2} \mathrm{O}$ and $100 \mathrm{ml}$ conc $\mathrm{H}_{2} \mathrm{SO}_{4}$ dissolved in the same amount water as the mixture), as suggested by A Heikkinen, Finland. Grass and wood samples are heated to ca $100^{\circ} \mathrm{C}$ for some hours with HCl, 1 to $2 \%$, left at least over night, washed with distilled water, transferred to $\mathrm{NaOH}, 1$ to $2 \%$, at $+80^{\circ} \mathrm{C}$ over night, washed with distilled water and finally acidified to $\mathrm{pH}<3$ before being dried. Combustion shortly follows pretreatment. Foraminifera and mollusk shells are leached with HCi. Fractions used for shell samples are given in per cent as a mean value. Since shell fragments usually are different in size, the fraction of shell samples does not give the fraction of individual shells.

Bone samples were treated to test different pretreatments (Olsson et al, 1974; El-Daoushy et al, 1978). Each method is indicated with the sample.

The reference sample is $95 \%$ of the ${ }^{14} \mathrm{C} /{ }^{12} \mathrm{C}$ ratio of the NBS oxalicacid standard. Any corrections for apparent water ages are thus not included here, but are discussed in papers dealing with the samples. Corrections for deviations from the normal ${ }^{13} \mathrm{C} /{ }^{12} \mathrm{C}$ ratio $(-25 \%$ in the PBD scale) are applied for unknown samples. Our 8 oxalic-acid samples did not show any significant difference according to measurements made in Stockholm (see also R, 1972, v 14, p 247).

The value, $5570 \mathrm{yr}$, has been used for the half-life of ${ }^{14} \mathrm{C}$. Results in this list are given BP (before 1950). Errors include standard deviation $(\sigma)$ of counted particles and uncertainties in corrections due to the ${ }^{13} \mathrm{C} /{ }^{12} \mathrm{C}$ ratio, filling pressure, temperature, working voltage, barometric pressure, etc, as described by Olsson (1966). When measured activity is lower than zero, $2 \sigma$ has been used for calculation of minimum age. When it is between zero and $2 \sigma$, net activity is increased by $2 \sigma$ for calculation of minimum age. Since results are physical measurements, no terms are included for the error in the half-life or previous ${ }^{1 \pm} \mathrm{C} / \mathrm{f}^{12} \mathrm{C}$ variations. These errors are cliscussed elsewhere.

Several samples had to be diluted with $\mathrm{CO}_{2}$ from an old source to bring them to normal working pressure of the counters. This has been ca $2000 \mathrm{mmHg}$ for most dates but ca 800 or $1300 \mathrm{mmHg}$ for some samples with numbers starting with U-5000 (PRF) measured with the new 
counter (PRF) described by El-Daoushy and Olsson (1977). Samples measured with Counter No. 1 have date numbers U-770-899 and -40004049; No. 4, U-2200-2299 and -2400-2810; No. 5 (same construction as PRF) U-4050-4300.

Description of samples are based on information provided by those who collected and submitted them.

\title{
ACKNOWLEDGMENTS
}

Special thanks are due $\mathrm{R}$ Ryhage and his co-workers for the ${ }^{13} \mathrm{C} /{ }^{12} \mathrm{C}$ determinations; $\mathrm{K}$ Siegbahn, who has made it possible to work at the institute; and Statens Naturvetenskapliga Forskningsråd, for financial support. The authors are indebted to Tomas Kronberg, Ciarina Sellin, Maud Södlerman and Birgitta Stenström for their laboratory work.

\section{SAMPI.E DESCIRIPTIONS}

\author{
I. GEOLOGIC SAMPLES
}

A. Canada

\section{U-2510. Cape Storm, Ellesmere I, North West Territories}

\author{
$9600 \pm 120$ \\ $\delta^{13} C=-17.2 \%$
}

Collagen received by EDTA method from Whale (probably Balaena mysticetus) from Cape Storm, Ellesmere I., NWT $\left(76^{\circ} 25^{\prime} \mathrm{N}, 87^{\circ} 33^{\prime} \mathrm{W}\right)$. Sample, part of mandible, embedded on raised beach, alt ca $118 \mathrm{~m}$. Coll 1970 by W Blake, Terrain Sc Div, Geol Survey Canada, Ottawa, Canada. Comment: sample dated by Canadian lab using method of Haynes (1967), GSCi-1496-A,B and C, $8900 \pm 60,9080 \pm 40,9260 \pm 45$ with $\delta^{13} \mathrm{C}$ values: $-16.2,-15.9$ and -15.4 , respectively. Sample also apatite clated according to Haynes (1968), GSC-1496-2, 8590 \pm 120 and $8860 \pm 65, \delta^{13} \mathrm{C}=$ $-13.5 \%$, using same gas in 2 counters (Blake, 1975). Uncertainty given by Blake red to give $\pm \sigma$ here. Other dates from Arctic Canada given and discussed by Blake (1975). Sample subm by Blake for interlab check.

\section{Amaroktalik, Bear Bay series}

Whale vertebra from Amaroktalik, Bear Bay, Cumberland Pen, Baffin I., NWT $\left(67^{\circ} 48^{\prime} 36^{\prime \prime} \mathrm{N}, 64^{\circ} 35^{\prime} 30^{\prime \prime} \mathrm{W}\right)$. Sample (CS-160874-1) consisted of chips in bad condition. Sample within beach boulder on surface of terrace, alt $10 \mathrm{~m}$. Coll 1974 and subm by C Schlüchter, Brock Univ, St Catharines, Ontario. Comment: 3 shell samples coll Aug 1974 from same locality to be clated at Brock Univ. Minor lichen growth seen. Sample believed to be ca $10,000 \mathrm{BP}$; thus, chosen by Uppsala for tests of different pre-treatment techniques. Although much later than thought dates of different fractions agree well.

\section{U-4144. Bear Bay, c: 1}

$$
\begin{array}{r}
1440 \pm \mathbf{5 0} \\
\delta^{1.3} C=-17.8 \% \text {. }
\end{array}
$$

Organic fraction obtained after EDTA treatment. Comment: gas obtained at clegassing and lst part of combustion. 
U-4143. Bear Bay, $c: 2$

$1250 \pm 50$

$\delta^{13} C=-17.5 \%$

Organic fraction obtained after EDTA treatment. Comment: same treatment as for U-4144 but gas from final part of combustion.

U.4141. Bear Bay, SOL, iso

$$
1310 \pm 50
$$

$\delta^{1.3} \mathrm{C}=-15.0 \%$

Organic fraction obtained after dissolving part of bone in 2- $\mathrm{N} \mathrm{HCl}$, neutralizing solution with $\mathrm{NaOH}$, using iso-electric point, dissolving precipitate in HCl to $\mathrm{pH}$ ca 3.5, heating during extraction, dialyzed.

U-4140. Bear Bay, INS, iso : 1

$$
\begin{array}{r}
1320 \pm 90 \\
\delta^{13} C=-17.6 \%
\end{array}
$$

Organic fraction obtained from parts not dissolved at initial HCl treatment of bone yielding U-4141. Solution after further HCl treatment neutralized with $\mathrm{NaOH}$, too little precipitate obtained. Liquid dried. Comment: gas obtained at degassing and 1st part of combustion.

\section{U-4139. Bear Bay, INS, iso:2}

$$
\begin{array}{r}
1280 \pm 70 \\
\delta^{1.3} C=-17.9 \%
\end{array}
$$

Organic fraction obtained after EDTA treatment. Comment: same treatment as for $\mathrm{U}-4140$ but gas from final part of combustion.

\section{Vestspitshergen series}

\section{B. Svalbard}

Shells and whale bones from Vestspitsbergen coll for determination of land uplift but also used for testing different methods for pretreatment of bones. Alt given above mean sea level. Previous determinations in this series in Radiocarbon: 1960, v 2, p 115-116; 1961, v 3, p 82-83; 1964, v 6, p 296-298; 1965, v 7, p $317-318 ; 1967$, v 9, p 456-457; 1969, v 11, p 521-524; 1972, v 14, p 255-257. Quaternary geology and land uplift disc by Birkenmajer (1960), Feyling-Hanssen (1955), Feyling-Hanssen \& Olsson (1959-1960), and Birkenmajer \& Olsson (1971). Samples from Hornsund coll 1970 and subm by K Birkenmajer, Polish Acad Sci, Krakow, and those from Isfjorden coll 1960 by D H Maling, R FeylingHanssen, Dept Geol, Aarhus Univ, Denmark, and I U Olsson. Comment: bone treatment with EDTA were shortly described (R, 1967, v 9, p 456) and in cletail by Olsson et al (1974).

\section{U-770. Wilczekodden E, 1st, Q7, B g, b}

Shell (Buccinum glaciale) from E coast of Wilczekodden $\left(77^{\circ} 00\right)^{\prime} \mathrm{N}$, $\left.15^{\circ} 56^{\prime} \mathrm{E}\right)$, Hornsund, Svalbard, alt $4 \mathrm{~m}$. Sample in shingle, fine, angular, 1 to $10 \mathrm{~cm}$ diam, Bed "a" of 1st Terrace, Loc 3 (Birkenmajer, 1960, p 21; fig 5). Comment: innermost $45 \%$ was used. 


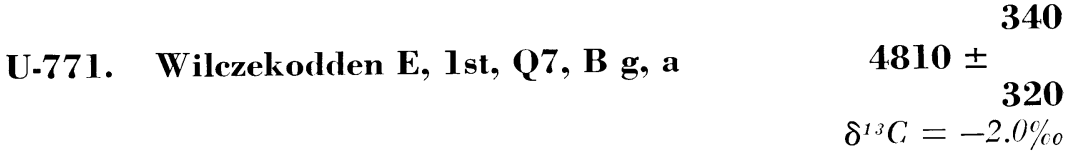

Shell layer surrounding U-770. Comment: layer corresponds to $50 \%$ of shell; $5 \%$ removed by washing.

\section{U-772. Wilczekodden E, Ist, Q7, M+S, b}

$$
\begin{array}{r}
4980 \pm \begin{array}{r}
210 \\
\mathbf{2 0 0}
\end{array} \\
\delta^{13} C=+1.7 \%
\end{array}
$$

Fragment (Mya truncata) 2.3g, and 3 shells (Saxicava arctica) 1.3g, from same bed as U-770, -771, -2251, -2240. Comment: innermost 55\% was used.

\section{U-773. Wilczekodden E, 1st, Q7, M+S, a}

$$
\begin{gathered}
4660 \pm \\
340 \\
\delta^{13} C=-0.3 \%
\end{gathered}
$$

Shell layer surrounding U-772. Comment: layer corresponds to $40 \%$ of shells; $5 \%$ removed by washing.

\section{U-2251. Wilczekodden E, 1st, Q7, B, b}

$$
\begin{array}{r}
\mathbf{4 7 4 0} \pm \mathbf{9 0} \\
\delta^{13} \mathrm{C}=-1.3 \%
\end{array}
$$

Fragments (Balanus sp) from same bed as U-770-773. Comment: innermost $30 \%$ was used.

U-2440. Wilczekodden E, 1st, Q7, B, a

$$
4820 \pm 120
$$

$\delta^{13} \mathrm{C}$ assumed $-1.3 \%$

Shell layer surrounding U-2551. Comment: layer corresponds to $35 \%$ of shells; $35 \%$ removed by washing.

U-782. Ishjørnhamna, 2a $\mathbf{a}_{2}, \mathbf{Q 2 a}, \mathbf{H C l},<\mathbf{2 5 0} \mu, \mathbf{r} \begin{array}{r}\mathbf{9 1 8 0} \pm \mathbf{3 7 0} \\ \delta^{1{ }^{3} C} \mathrm{C}=-19.0 \%\end{array}$

Insoluble remains after HCl treatment of whalebone from Fuglebergsletta, Isbjornhamna $\left(77^{\circ} 00^{\prime} \mathrm{N}, 15^{\circ} 33^{\prime} 30^{\prime \prime} \mathrm{E}\right), 82 \mathrm{~m}$ SW Polish Sta, Hornsund, Svalbard, alt $8 \mathrm{~m}$. Sample from lower jaw, $1.9 \mathrm{~m}$ long, $17 \mathrm{~cm}$ diam, 2/3 buried in tundra: mosses, lichens, Saxifraga, Salix polaris, and grass, $2 \mathrm{a}$.. Terrace. Comment: grains $<250 \mu$ used. $\mathrm{pH}$ of extracts same as $\mathrm{HCl}$ used for last 10 to 12 extractions (18 days); ca 30 extractions. 0.1-N HCl used. Bone dated previously (R, 1969, v 11, p 523) using more reliable EDTA-treatment.

U.783. Isbjørnhamna, 2a 2 , Q2a, HCl, $<250 \mu, 1 \quad 4190 \pm 830$

First 9 extracts from HCI treatment of bone also used for U-78? Comment: liquid purified by dialysis. 


\section{U-778. Kapp Linné 6005, A2, R}

Organic fraction of vertebra from Kapp Linné $\left(78^{\circ} 04^{\prime} \mathrm{N}, 13^{\circ} 40^{\prime} \mathrm{E}\right)$, Isfjorden, Svalbard, alt ca $1 \mathrm{~m}$. Same vertebra as for U-2270. Comment: acetone-treated before EDTA treatment. Diluted.

U-758. Kapp Linné 6005, A2, A

$$
\begin{aligned}
& 3700 \\
& 8700 \pm \\
& \delta^{13} \mathrm{C}=-25.6 \%
\end{aligned}
$$

Acetone extract from bone used for U-778. Comment: probably contaminated with oil from radio sta. Diluted.

U-779. Kapp Linné 6005, A2, W

$$
\begin{array}{r}
140 \pm 140 \\
\delta^{13} \mathrm{C}=-25.9 \%
\end{array}
$$

Wrong fraction of same vertebra as used for U-758. Diluted.

U-777. Kapp Linné, A1, W diluted.

Wrong fraction of same vertebra as used for U-2270. Comment:

\section{U-785. Kapp Linné, 6005, C1, SOL}

$$
\begin{array}{r}
480 \pm 100 \\
\delta^{1.3} C^{C}=-18.1 \%
\end{array}
$$

Soluble fraction of same vertebra as $\mathrm{U}-778$, received after $\mathrm{HCl}$ treatment. Comment: soluble fraction dialyzed.

U-786. Kapp Linné 6005, C1, INS

$$
\begin{array}{r}
\mathbf{3 3 0} \pm \mathbf{1 3 0} \\
\delta^{1.3} C=-16.6 \%
\end{array}
$$

Insoluble remains after treatment to get U-785. Comment: diluted.

\section{U-774. Gipshuken 6016 c:1}

$5000 \pm 160$

$\delta^{1.3} \mathrm{C}=-17.8 \%$

Organic fraction of whalebone from Gipshuken $\left(78^{\circ} 27^{\prime} \mathrm{N}, 16^{\circ} 24^{\prime}\right.$ E), Isfjorden, Svalbard, alt $10 \mathrm{~m}$. Comment: EDTA treatment. Gas obtained at degassing and lst part of combustion.

\section{U-775. Gipshuken 6016 c:2}

$4980 \pm 120$

$\delta^{13} \mathrm{C}=-17.0 \%$

Organic fraction of same bone as used for U-774. Comment: gas obtained at final part of combustion.

\section{U-784. Gipshuken $6016 \mathrm{c} \mathrm{W}$}

Wrong fraction of same whalebone as used for U-774 and -775.

\section{U-2538. Gipshuken 6017 c:1}

$5430 \pm 65$ $\delta^{13} \mathrm{C}=-17.5 \%$

Organic fraction of whalebone from Gipshuken, alt $12 \mathrm{~m}$. Comment: EDTA treatment including dialysis of extracted collagen. 


\section{U-2546. Gipshuken 6017 c:1 W}

$-830 \pm 110$

$\delta^{1.3} \mathrm{C}=-21.1 \% \mathrm{c}$

Wrong fraction received at pretreatment of U-2538. Comment: diluted.

\section{U-4056. Gipshuken $6017 \mathrm{c}: 2$}

$$
6370 \pm \begin{aligned}
& 1250 \\
& \delta^{1.3} C=-23.7 \%(c 0
\end{aligned}
$$

Organic fraction of outer very porous parts, which normally should have been rejected, of same whalebone as used for U-2538. Comment: EDTA treatment including dialysis. Fraction soluble in cool water, pH ca 6. Diluted.

\section{U-4057. Gipshuken $6017 \mathrm{c}: 2 \mathrm{R}+\mathrm{W}$}

$$
\begin{array}{r}
\mathbf{2 8 6 0} \pm \mathbf{8 0} \\
\delta^{1.3} C=-26.3 \%
\end{array}
$$

Wrong fraction mixed with parts of right fraction of same piece of bone as used for U-4056.

\section{U-879. Gipshuken 6017 c:3}

$$
\begin{array}{r}
\mathbf{8 3 4 0} \pm \underset{670}{660} \\
\delta^{1.3} \text { C assumed }-19.0 \% \%
\end{array}
$$

Impure organic fraction of same whalebone as used for U-2538. Comment: Experiment by removing EDTA by dialysis of gelatinous sample. Not all EDTA removed. Date should not be used. Diluted.

\section{U-2537. Gipshuken 6017 b:1a}

$$
\begin{array}{r}
\mathbf{5 5 5 0} \pm \mathbf{1 0 0} \\
\delta^{1.3} \mathrm{C}=-18.0 \% \%
\end{array}
$$

Organic fraction of same whalebone as used for U-2538. Comment: HCil treatment followed by neutralization with $\mathrm{NaOH}$. Insoluble fraction from this treatment clissolved in HCil, pH ca 4. Solution purified by dialysis.

\section{U-4051. Gipshuken 6017 b:2a}

$$
\begin{array}{r}
1220+160 \\
\delta^{1.3} \mathrm{C}=-24.0 \%
\end{array}
$$

Organic fraction of rather porous parts, which normally should have been rejected, of same whalebone as used for U-2538. Comment: HCI treatment. Soluble fraction used after dialysis. I)iluted.

\section{U-4032. Gipshuken $6017 \mathrm{~b}: 1 \mathrm{~b}$}

$$
\begin{array}{r}
1620 \pm \quad 380 \\
\mathbf{3 7 0} \\
\delta^{1.5} \mathrm{C}=-17.5 \%
\end{array}
$$

Insoluble remains after HCil treatment to get U-2537. Comment: further extraction by HCil at elevated temperature. Neutralization with $\mathrm{NaOH}, \mathrm{pH}$ ca 6.6, precipitate clialyzed, HCil added until pH 3 to 4, 
heated, soluble fraction dialyzed, evaporated down and sample extracted at room temperature and $\mathrm{pH}$ ca 2.

\section{U-4052. Gipshuken 6017 b:2b}

$1730 \pm 100$

$\delta^{13} \mathrm{C}=-22.5 \%$

Insoluble fraction after $\mathrm{HCl}$ treatment to get U-4051. Comment: further washing in dialysis bag, dissolved in water, $\mathrm{pH}$ ca 5. Diluted.

\section{U.4036. Gipshuken 6017 a:1 inorg}

$3935 \pm 85$

$\delta^{13} \mathrm{C}=-14.4^{\mathrm{C}} / \mathrm{co}$

Apatite CO.2 obtained after treatment of bone used for U-2538 with acetic acid for 2 clays at slightly elevated temperature, careful washings with water for 2 weeks. $\mathrm{CO}_{2}$ released by $\mathrm{HCl}$ in stream of oxygen.

\section{U-4050. Gipshuken 6017 a:2 inorg \\ $\delta^{13} \mathrm{C}=-15.2 \%$}

Apatite $\mathrm{CO}$ obtained after treatment of rather porous parts of whalebone used for U-2538 with acetic acid for 2 days at slightly elevated temperature, with new acetic acid under vacuum for 11 clays, and careful washings with water. $\mathrm{CO}_{2}$ released by $\mathrm{HCl}$ in stream of oxygen. Comment: diluted.

\section{U-4033. Gipshuken 6017 a:1 s}

$$
\begin{aligned}
& 4780 \pm \\
& 270 \\
& \delta^{1.3} \mathrm{C}=-19.6 \%
\end{aligned}
$$

Soluble organic fraction obtained at HCil treatment to get U-4036. Comment: impurities removed by precipitation with $\mathrm{NaOH}, \mathrm{pH} 3$ to 4 , liquid reduced in volume, dialyzed, evaporated down and used. Diluted.

\section{U-4053. Gipshuken 6017 a:2 s}

$$
\begin{array}{r}
4050 \pm \underset{1180}{1390} \\
\delta^{13} C=-27.3 \%
\end{array}
$$

Soluble organic fraction obtained at HCI treatment to get U-4050. Comment: liquid reduced in volume, purified by dialysis and used. Diluted.

\section{U-4034. Gipshuken 6017 a:1 ha}

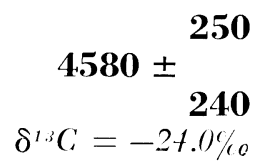

In $\mathrm{NaOH}, 1 \%$, soluble fraction of insoluble remains obtained at HCl treatment to get U-4036 and -4033. Comment: sample precipitated with HCil as for humic acid. Diluted. 
U-4054. Gipshuken 6017 a:2 ha

In $\mathrm{NaOH}, 1 \%$, soluble fraction of insoluble remains obtained at HCl treatment to get U-4050 and -4053. Comment: sample precipitated with $\mathrm{HCl}$ as for humic acid. Diluted.

\section{U-4035. Gipshuken 6017 a:1 INS}

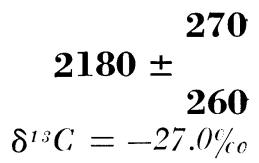

Insoluble remains, washed and made acid, obtained at treatment to get U-4034. Comment: diluted.

U-4055. Gipshuken 6017 a:2 INS

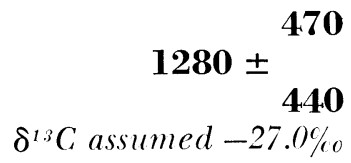

Insoluble remains, washed and made acid, obtained at treatment to get U-4054. Comment: diluted.

\section{U-4058. Gipshuken $6017 \mathrm{~W}$}

$1805 \pm 75$

$\delta^{1 .} \mathrm{C}$ assumed $-25.0 \%$

Fraction released at ultrasonic washing and boiling in water. Comment: slightly acidified.

\section{U-4059. Gipshuken 6017 hpe SOL}

$$
\begin{array}{r}
4110 \pm \begin{array}{r}
460 \\
440
\end{array} \\
\delta^{13} C=-22.7 \%
\end{array}
$$

Fraction removed physically from ground samples used for dating. Comment: sample may contain pieces of bone as well as contaminants deriving from outer porous and very porous parts usually removed from samples before dating. Fraction soluble in HCl.

U-4064. Gipshuken 6017 hpe INS

$$
\begin{array}{r}
1820 \pm \\
400 \\
\delta^{13} C=-27.9 \% \\
\mathbf{3 8 0}
\end{array}
$$

Insoluble remains obtained at treatment to get U-4059. Comment: diluted.

\section{U-2436. Ekholmvika 6022, c 71}

$$
9710 \pm 100
$$

$\delta^{1.3} \mathrm{C}=-17.1 \%$

Whale rib from Ekholmvika $\left(78^{\circ} 35^{\prime} \mathrm{N}, 16^{\circ} 38^{\prime} \mathrm{E}\right)$, Billefjorden, Svalbard; alt ca $50 \mathrm{~m}$. Deeply buried in fine gravel. Coll 1969 by D H Maling. Comment: EDTA treatment. 


\section{U-2406. Ekholmvika 6022, b HCl, $<500 \mu \mathrm{m}$, SOL, H.O SOL}

$9680 \pm 90$
$\delta^{1 .} C=-15.6 \%$

Same whale rib as for U-2436. Fraction $<500 \mu \mathrm{m}$ ground to fine powder. Treated with $2-\mathrm{N} \mathrm{HCl}$, left in acid for $11 / 2 \mathrm{mos}$, filtrated, liquid evaporated, dissolved in water.

\section{U-2407. Ekholmvika 6022, b HCl, $<500 \mu \mathrm{m}$, INS, HO SOL}

$$
\begin{array}{r}
9600 \pm 90 \\
\delta^{13} C=-17.4 \%
\end{array}
$$

Same whale rib as for U-2406. Same treatment but fraction not dissolved by acid used. Extracted by acidic water.

\section{Norway}

\section{U-2766. Toten tusk}

U-4214.

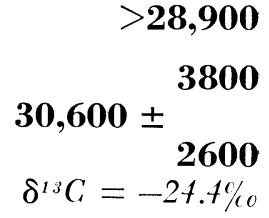

Collagen, obtained by EDTA method, but H.O soluble and insoluble fractions, from tusk of Mammutus primogenius from Skreia, Oppland Co $\left(60^{\circ} 3912^{\prime} \mathrm{N}, 10^{\circ} 551 / 2^{\prime} \mathrm{E}\right)$. Sample from $1.5 \mathrm{~m}$ below surface in moraine in ditch $2 \mathrm{~m}$ deep (Heintz, 1956). Coll 1955, subm 1976 by $\mathrm{N}$ Heintz, Palentol Mus, Oslo Univ to Follestad, Norges geol unders $\phi$ kelse, Trondheim, for age determination in Uppsala. Comment: intrusion of water-soluble compounds studied by Follestad (1977). Sample, 150g, from hard part. Porous parts of tusk dated 1962 by Nydal, T-359, 19,190 \pm 1200. Sample, only $30 \mathrm{~g}$, was too small for thorough pretreatment and date regarded as too low because of contamination. Unpub by Nydal (pers commun) although used by others. Heintz (1965) used result to date mammoth from before end of last glaciation. New determination is underway in Trondheim. Present sample, pretreatment, age calculation and result $\left(\right.$ mean value $\left.32,100 \pm \begin{array}{r}3100 \\ 2300\end{array}\right)$ discussed by Follestad and Olsson (1979). Diluted.

\section{Varangerfjord series}

Whale rib from Makviken (Nesseby), Varangerfjord, Norway $\left(70^{\circ}\right.$ $2^{\prime} 20^{\prime \prime} \mathrm{N}, 29^{\circ} 07^{\prime} 43^{\prime \prime}$ E). Sample from accumulation of shelly gravel and sand, 15.8m above present Balanus line (Donner et al, 1977). Subm 1975 by J Donner, Dept Geol Palaeontol, Univ Helsinki, Finland. Comment: Mytilus edulis shells from same accumulation, regarded as reliable clated as Hel-624, 4120 $\pm 130, \delta^{13} \mathrm{C}=+1.7 \%$, but not normalized to $\delta^{13} \mathrm{C}=$ $-25 \%$. After normalization radiocarbon age is $4560 \mathrm{yr}$ without correction for reservoir effect. Mya truncata from same layer dated as Hel-625, 
$6430 \pm 150$, but not regarded as suitable for land uplift dating (Donner et $a l, 1977)$. Land uplift here corresponds to $1 \mathrm{~m}$ in $160 \mathrm{yr}$.

U.4125. Varangerf jord, Nesselby, c: 1

$$
5140 \pm 170
$$

$\delta^{1.3} C=-17.2 \%$

Organic fraction obtained after EDTA treatment. Comment: gas obtained at degassing and lst part of combustion.

\section{U-4126. Varangerfjord, Nesseby, c:2}

$$
\begin{array}{r}
4710 \pm \mathbf{1 5 0} \\
\delta^{13} C=-16.6 \%
\end{array}
$$

Organic fraction obtained after EDTA treatment. Comment: same treatment as for U-4125 but gas from final part of combustion.

U-4128. Varangerf jord, Nesseby, SOL, iso

$$
4095 \pm 100
$$

$\delta^{1.3} \mathrm{C}=-16.7 \%$

Organic fraction obtained after clissolving part of bone in 2-N HCl, neutralizing solution with $\mathrm{NaOH}$, using iso-electric point, dissolving precipitate in $\mathrm{HCl}$ to $\mathrm{pH} 3.0$ to 3.5 , heating during this extraction, dialysis.

U-4127. Varangerfjord, Nesseby, INS, iso

$$
\begin{array}{r}
4320 \pm 320 \\
4085 \pm 190 \\
\delta^{13} C=-12.5 \%
\end{array}
$$$$
\text { U-2751. }
$$

Organic fraction received from parts not dissolved at initial HCl treatment of bone yielding U-4128. Solution alter further HCl treatment neutralized with $\mathrm{NaOH}$, precipitate dialyzed and sample then treated as $\mathrm{U}-4128$.

\section{Ageröd series}

\section{Sweden}

Collagen from bone fragments of red deer from white layer in raised bog, Ageröds mosse, Munkarp parish, Skåne, Sweden (55 $5612^{\prime} \mathrm{N}, 13^{\circ}$ 25' E). Subm 1974 by $\mathrm{S}$ Håkansson, Radiocarbon Dating Lab, Dept Quaternary Geol, Univ Lund, after grinding. Other half of sample dated by Håkansson, Lu-872, 7220 $\pm 70, \delta^{13} \mathrm{C}=-22.9 \%$ (Håkansson, 1976).

\section{U-4081. Ageröd I:HC, Sample 6, CN}

$$
\begin{array}{r}
\mathbf{7 7 5 0} \pm \mathbf{8 0} \\
\delta^{1.3} C=-22.9 \%
\end{array}
$$

Collagen obtained after EDTA treatment (Olsson et al, 1974), but fraction soluble in cool water, neutral soluble, used. Diluted.

\section{U-4082. Ageröd I:HC, Sample 6, WA}

$7415 \pm 115$

$\delta^{1.3} \mathrm{C}=-23.5 \%$

Collagen obtained after same treatment as U-4081, but fraction extracted after U-4081 using hot slightly acid water used. Diluted.

General Comment: mean value of present results lower than other results from Ageröd I:HC except Lu-872 (Håkansson, 1976). 


\section{Dra Abu El-Naga series}

\section{A. Egypt}

Samples from Ina Abu El-Naga S, on W bank of Nile R opposite Luxor, Egypt $\left(25^{\circ} 43^{\prime} \mathrm{N}, 32^{\circ} 38^{\prime} \mathrm{E}\right)$. Samples from pyramidal tomb superstructures, built of courses of mud brick separated by beddings of halfa grass with occasional use of timber reinforcement. Coll April 13 to 15, 1970 by Lanny Bell, Univ Mus, Univ Pennsylvania, Philadelphia, USA. Subm by E Ralph and H N Michael, Univ Mus, Univ Pennsylvania as interlaboratory check and joint project. Description of provenience by Fishman et al (R, 1977, v 19, p 198). No details concerning provenience given directly to Uppsala. Samples classified as primary, secondary or tertiary samples by Bell. Historic ages related to Ramses II. His reign supposedly started in $1290 \mathrm{BC}$. Historical ages are: Nebwenenef, Tomb 157. High Priest, 1290-1273 BC; Bekenkhons I, Tomb 35, High Priest, 1273-1223 BC; Roma-Roy, Tomb 283, High Priest, 1244-1196 вс; 'Tjanefer, Tomb 158, 1267-1168 BC; Inhernakht, called Nakhtmin by Fishman et al, Tomb 282, Chief of Bowmen of Kush, 1290-1223 Bc. Ages as given by T Säve-Södlerbergh, Uppsala Univ, Uppsala, Sweden (pers commun). Small portions of reed samples id by Vivi Täckholm, Bot Dept, Fac Sci, Cairo Univ, Giza, Egypt. Portions of bulk reed samples also id by D F Cutler, Jodrell Lab, Royal Bot Gardens, Surrey, England. Wood samples id by B F Kukachka, Forest Prod Lab, US Dept Agric, Madison, Wisconsin. Details concerning treatment and results given by Olsson and El-Daoushy (1978) except 2 samples for which same gas was re-dated and another 2 samples for which $\mathrm{NaOH}$ soluble fraction was dated.

General Comment: all samples of Desmostachya bipinnata (L) Stapf, have yielded $\delta^{13} \mathrm{C}$ values between -9.8 and $-12.4 \%$, each with an uncertainty of ca $0.5 \%$, for $\mathrm{NaOH}$ insoluble fraction. This value is typical for $\mathrm{C}_{4}$ plants. Samples dated at Pennsylvania, pretreated with HCl but not with $\mathrm{NaOH}(\mathrm{R}, 1977$, v 19, p 188), show a strange spread in their $\delta^{13} \mathrm{C}$ values compared with present results. Comparison of results for U-862, -2462, -5002, -2800, -869, -2469, -5004, -870, P-1732, -1739, -1441, and BN-339 implies that insoluble fractions of tertiary reeds from Tomb 157 and primary reeds from Tomb 35 might have been interchanged. New measurements underway indicate that this is the case.

U-880. Nebwenenef, Dyn XIX, P R

$2815 \pm 140$

U-2460.

$$
2970 \pm 80
$$

U-5000.

$2865 \pm 110$

$\delta^{1 .} C=-21.2 \%$

Primary reeds, Desmostachya bipinnata (L) Stapf and Phoenix dactylifera, from pyramid of Nebwenenel. Commenl: sample dated as Phoenix dactylifera, P-1730, $3120 \pm 50, \delta^{1: C} \mathrm{C}=-19.1 \%$, and $\mathrm{P}-1730-\mathrm{A}$, $3210 \pm 50, \delta^{1:} \mathrm{C}=-15.7 \%$. 
U-881. Nebwenenef, Dyn XIX, S R

$3060 \pm 125$

U-2461.

$3050 \pm 60$

U-5001.

$3240 \pm 110$

$\delta^{13} \mathrm{C}=-10.6 \%$

Secondary reeds, Desmostachya bipinnata (L) Stapf, from pyramid of Nebwenenef. Comment: sample dated as (?) Desmostachya bipinnata, P-1731, $3010 \pm 60, \delta^{13} \mathrm{C}=-11.1 \%$. Another secondary sample, same species, P-1825, $2940 \pm 50, \delta^{13} \mathrm{C}=-18.0 \%$, also BM-658b.

U-862. Nebwenenef, Dyn XIX, T R

$$
\begin{array}{r}
1390 \pm 135 \\
1360 \pm 60 \\
1480 \pm 110 \\
\delta^{13} C=-10.9 \%
\end{array}
$$$$
\text { U-2462. }
$$

U-5002.

Tertiary reeds, Desmostachya bipinnata (L) Stapf, from pyramid of Nebwenenef. Comment: sample dated as (?) Desmostachya bipinnata, $\mathrm{P}-1732,3030 \pm 60, \delta^{13} \mathrm{C}=-11.9 \%$.

U-2800. Nebwenenef, Dyn XIX, T R SOL

$$
\begin{array}{r}
\mathbf{2 6 3 0} \pm \mathbf{1 9 0} \\
\delta^{13} \mathrm{C}=-16.5 \%
\end{array}
$$

In $\mathrm{NaOH}$ soluble fraction from pretreatment yielding U-862, -2462 and -5002 . Fraction SOL yielded $1 / 5$ amount of gas from insoluble fraction.

\section{U-869. Bekenkhons I, Dyn XIX, P R}

$$
\begin{array}{r}
2965 \pm 135 \\
2975 \pm 70 \\
2975 \pm 70 \\
\delta^{13} C=-10.7 \%
\end{array}
$$$$
\text { U-2469. }
$$

U-5004.

Primary reeds, Desmostachya bipinnata (L) Stapf, from pyramid of Bekenkhons I. Comment: sample dated as (?) Desmostachya bipinnata, P-1739-A, $1660 \pm 50, \delta^{13} \mathrm{C}=-1.4 \%$. Another sample coll by Martin dated as P-1441, $1120 \pm 40$ and as BMI-339, $1210 \pm 110(\mathrm{R}, 1971, \mathrm{v} 13$, p 162). A 3rd sample, (?) Desmostachya bipinnata, dated as secondary sample, P-1440, $3000 \pm 60, \delta^{13} \mathrm{C}=-19.0 \%$.

\section{U-870. Bekenkhons I, Dyn XIX, P R SOL}

$$
\begin{array}{r}
1420 \pm 80 \\
\delta^{13} C=-15.9 \%
\end{array}
$$

In $\mathrm{NaOH}$ soluble fraction from pretreatment yielding U-869. Fraction SOL yielded $1 / 3$ amount of gas from insoluble fraction.

$$
\begin{array}{rrr}
\text { U-2495. } & \text { Roma-Roy, Dyn XIX, P R } & 2915 \pm 80 \\
\text { U-5005. } & 2940 \pm 80 \\
& \delta^{13} C=-12.4 \%
\end{array}
$$

Primary reeds, Desmostachya bipinnata (L) Stapf, from pyramid of Roma-Roy. Comment: sample dated as (?) Desmostachya bipinnata, $\mathrm{P}-1735,3130 \pm 40, \delta^{13} \mathrm{C}=-9.6 \%$. 
U-5016. Roma-Roy, Dyn XIX, S R

$2595 \pm 105$

$\delta^{13} \mathrm{C}=-11.5 \%$

Secondary reeds, Desmostachya bipinnata (L) Stapf, from pyramid of Roma-Roy. Comment: same gas re-dated later as U-4166 and -5048. Sample dated as (?) Desmostachya bipinnata, P-1736, $3280 \pm 50, \delta^{13} \mathrm{C}=$ $-6.6 \%$.

\section{U-4166. Roma-Roy, Dyn XIX, S R}

U-5048.

Same gas as for $\mathbf{U}-5016$ re-measured.

\section{U-2497. Roma-Roy, Dyn XIX, W 1}

U-5017.

$$
\begin{array}{r}
2680 \pm 95 \\
2670 \pm 140 \\
\delta^{1.3} C=-11.5 \%
\end{array}
$$

$$
\begin{array}{r}
2925 \pm 80 \\
3005 \pm 110 \\
\delta^{1.3} \mathrm{C}=-30.8 \% \%
\end{array}
$$

Tamarix wood from branch protruding from pyramid of Roma-Roy. Comment: sample dated as P-1737, $3120 \pm 50, \delta^{13} \mathrm{C}=-25.7 \%$, P-1737-A, $3020 \pm 80, \delta^{13} \mathrm{C}=-26.6 \%$. Same wood dated, according to Fishman et al (1977), as BM-338, $3030 \pm 85, \delta^{13} \mathrm{C}=-28.3 \%(\mathrm{R}, 1971, \mathrm{v} 13, \mathrm{p} 162)$ and UCLA-1395, $2880 \pm 60, \delta^{13} \mathrm{C}=-25.2 \%$ (Berger, 1970).

\section{U-5018. Roma-Roy, Dyn XIX, W 2}

$$
\begin{array}{r}
\mathbf{2 6 4 0} \pm \mathbf{1 3 5} \\
\delta^{1.3} \mathrm{C}=-25.8 \%
\end{array}
$$

Tamarix wood from branch embedded in pyramid of Roma-Roy. Comment: same gas re-dated later as U-2693 and U-5049. Sample dated as U-2498 and U-5019 after new pretreatment, P-1738, $3060 \pm 50, \delta^{13} \mathrm{C}=$ $-25.7 \%$.

\section{U-2498. Roma-Roy, Dyn XIX, W 2}

$$
\begin{array}{r}
\mathbf{3 1 7 0} \pm \mathbf{7 0} \\
\mathbf{2 7 3 5} \pm \mathbf{1 4 0} \\
\delta^{1.3} C=-26.3 \%
\end{array}
$$

U-5019.

Same Tamarix wood as used for U-5018 but new pretreatment.

\section{U-2693. Roma-Roy, Dyn XIX, W 2}

$$
\begin{array}{r}
3020 \pm 70 \\
2760 \pm 150
\end{array}
$$

U-5049.

Same gas as for U-5018 re-measured.

\section{U-866. Tjanefer, Dyn XX, P R}

$$
\begin{array}{r}
\mathbf{2 8 0 5} \pm \mathbf{9 0} \\
\delta^{1.3} \mathrm{C}=-11.1 \% \mathrm{c}
\end{array}
$$

Primary reeds, Desmostachya bipinnata (L) Stapf, from pyramid of Tjanefer. Comment: sample dated as Desmostachya bipinnata, P-1696, $3080 \pm 60, \delta^{13} \mathrm{C}=-13.4 \%$. Other reed samples coll by Martin dated as BM-396, $2890 \pm 100, \delta^{13} \mathrm{C}=-14.4 \%$ and UCLAA-1393, $3060 \pm 60, \delta^{13} \mathrm{C}=$ $-11.3 \%$ (R, 1971, v 13, p 162; Berger, 1970).

U-868. Tjanefer, Dyn XX, S R

$2800 \pm 80$ 
U-5028.

$\delta^{13} \mathrm{C}=-11.3 \%$

Secondary reeds, Desmostachya bipinnata (L) Stapf, from pyramid of Tjanefer. Comment: sample dated as primary sample Desmostachya bipinnata, P-1698, $2990 \pm 50, \delta^{13} \mathrm{C}=-13.4^{\prime} / \%$.

U-2499. Tjanefer, Dyn XX, W $2910 \pm 70$

U-5029.

Acacia wood from branch protruding from pyramid of Tjanefer. Comment: sample dated as P-1699, $3010 \pm 50, \delta^{13} \mathrm{C}=-25.7 \%$. Wood coll by Martin dated as $\mathrm{BN}-337,3080 \pm 75, \delta^{13} \mathrm{C}=-26.9 \%$ o $(\mathrm{R}, 1971$, $\mathrm{v} 13, \mathrm{p} 162)$. One wood sample dated as UCLA-1394, $3030 \pm 60, \delta^{13} \mathrm{C}=$ $-24.0 \%$ (Berger, 1970).

\section{U-863. Inhernakht, Dyn XIX, P R 1}

$$
\begin{array}{r}
2830 \pm 95 \\
2690 \pm 120 \\
\delta^{13} C=-11.0 \%
\end{array}
$$$$
\text { U-5013. }
$$

Primary reeds, Desmostachya bipinnata (L) Stapf, from within pyramid of Inhernakht. Comment: sample dated as U-2803 after new pretreatment, (?) Desmostachya bipinnata, P-1733, $2920 \pm 50, \delta^{13} \mathrm{C}=-3.9^{1} \%$.

\section{U-2803. Inhernakht, Dyn XIX, P R 2}

$$
\begin{array}{r}
3020 \pm 110 \\
\delta^{13} C=-10.2 \%
\end{array}
$$

Same reed sample as used for U-863 and -5013 but new pretreatment, 2 extractions with $\mathrm{NaOH}$.

\section{U-864. Inhernakht, Dyn XIX, S R 1}

$$
\begin{array}{r}
2855 \pm 115 \\
\mathbf{2 3 7 0} \pm 120 \\
\delta^{1.3} C=-11.9 \%
\end{array}
$$$$
\text { U-5014. }
$$

Secondary reeds, Desmostachya bipinnata (L) Stapf, from pyramid of Inhernakht. Comment: sample clated as U-2802 after new pretreatment, and (?) Desmostachya bipinnata, P-1734, 3400 $\pm 60, \delta^{13} \mathrm{C}=-8.3 \%$, P-1734-A, $3940 \pm 50, \delta^{13} \mathrm{C}=-3.0 \%$.

\section{U-2801. Inhernakht, Dyn XIX, S R I SOL}

$$
\begin{array}{r}
3110 \pm 170 \\
\delta^{15} C=-13.3 \%
\end{array}
$$

In $\mathrm{NaOH}$ soluble fraction from pretreatment yielding U-864 and -5014 . Fraction SOL yielded $40 \%$ of amount of gas from insoluble fraction.

\section{U-2802. Inhernakht, Dyn XIX, S R 2}

$$
\begin{array}{r}
3090 \pm 110 \\
\delta^{13} C=-9.8 \%
\end{array}
$$

Same reed sample as used for U-864 and -5014 but new pretreatment, 2 extractions with $\mathrm{NaOH}$. 
III. RESERVOIR EFFECT

\section{Western Sweden series}

U-4142. Morup 2097, c

$$
\begin{array}{r}
415 \pm 45 \\
\delta^{13} C=-12.9 \%
\end{array}
$$

Balaenoptera physalus along coast at Morup, $7 \mathrm{~km}$ long, Halland, Sweden $\left(56^{\circ} 59^{\prime} \mathrm{N}, 12^{\circ} 24^{\prime} \mathrm{E}\right)$. Coll Nov 241875 , subm to Naturhist mus in 1903 and subm to ${ }^{14} \mathrm{C}$ lab by C Fredén, Sveriges Geol Undersökning, Göteborg. Comment: EDTA treated. Another part of same bone, also EDTA treated, had $\delta^{13} \mathrm{C}=-14.5 \%$, but this part was lost. Liquid from same EDTA-treated bone but from initial boiling in water was recovered and measured as $\mathrm{U}-2650$.

\section{U-2650. Morup 2097, $\mathrm{H}_{2} \mathrm{O}$}

$$
\begin{array}{r}
\mathbf{2 0 0} \pm \mathbf{3 8 0} \\
\delta^{13} \mathrm{C} \text { assumed }-17.0 \% / \%
\end{array}
$$

Water extract from initial treatment of bone before EITA treatment.

\section{U-4168. Lysekil 437,}

$$
\begin{array}{r}
360 \pm 100 \\
\delta^{13} C=-12.5 \%
\end{array}
$$

Orcinus orca from close to Lysekil, Bohuslän, Sweden $\left(58^{\circ} 17^{\prime} \mathrm{N}\right.$, $11^{\circ} 26^{\prime} \mathrm{E}$ ), killed Dec 18, 1868 and subm to Naturhist mus. Subm by J Iepiksaar, Naturhist mus, Göteborg to Fredén to forward it to ${ }^{14} \mathrm{C}$ lab. 1)escription by Malm (1871, p 79). Comment: EDTA treated.

\section{Eastern Sweden series}

\section{U-4211. Nätrafjärden, Älgön, c:1}

$$
\begin{array}{r}
\mathbf{6 5 0} \pm \mathbf{5 5} \\
\delta^{1.3} \mathrm{C}=-18.2 \%
\end{array}
$$

Whale from Nätrafjärden, Angermanland, Sweden $\left(6314^{\circ} \mathrm{N}, 1812^{\circ}\right.$ E). Whale trapped in winter 1657 and found dead in 1658 (Nordlander, 1934). Subm to the ${ }^{14} \mathrm{C}$ lab with aid of Ake Hörnsten, Sveriges Geol Undersökning, Stockholm and Fredén. Comment: EDTA treated. Gas obtained at degassing and 1st part of combustion.

\section{U-4167. Nätrafjärden, Älgön, c:2}

$$
\begin{array}{r}
\mathbf{6 4 0} \pm \mathbf{5 5} \\
\delta^{13} \mathrm{C}=-17.0 \%
\end{array}
$$

EDTA-treated but gas obtained at final part of combustion. Comment: expected ${ }^{14} \mathrm{C}$ age ca $200 \mathrm{yr}$.

\section{U-4113. Caspian Sea 4614 c}

$\mathbf{4 5 5} \pm \mathbf{5 0}$

$$
\delta^{13} \mathrm{C}=-15.4 \%
$$

Phoca caspica from Kulalai, Caspian Sea $\left(45^{\circ} \mathrm{N}, 50^{\circ} \mathrm{E}\right)$. Coll 1899 , subm to Naturhist Riksmus, Stockholm by Lönnberg. Rear extremity used. Subm to ${ }^{1+} \mathrm{C}$ lab by $\mathrm{G}$ Westergren. Comment: EDTA treated.

\section{U-4219. Trosa 7771, c}

$$
\delta^{13} \mathrm{C}=-22.0 \%
$$

Alces alces from close to Trosa, (ca $58^{\circ} 55^{\prime} \mathrm{N}, 1712^{\circ}$ E) Sweden. Coll 1881. Sample bought through Nordenskiöld, subm to Naturhist 
Riksmus. Subm to ${ }^{14} \mathrm{C}$ lab by Westergren to date sample free from reservoir effect of sea. Comment: EDTA treated.

\section{Tromsö whaling station series}

Whales hunted June 1971. Subm by Stig Skreslet, Narinbiol stasjon, Troms $\phi$, Norway. Comment: bones treated with acetone before EDTA treatment and collagen as well as extract dated (Olsson et al, 1974).

\section{U-878. Fin whale 1 c}

Collagen fraction.

$$
\delta^{13} \mathrm{C}=-18.6 \%
$$

\section{U-2467. Fin whale 1 f}

$$
\begin{array}{r}
-605 \pm 60 \\
\delta^{13} C=-18.6 \%
\end{array}
$$

Extract obtained by acetone treatment before EDTA treatment to yield collagen for U-878. Acetone carefully removed.

\section{U-2466. Fin whale 2 le}

$$
\begin{array}{r}
-150 \pm 60 \\
\delta^{13} C=-16.0 \% / \%
\end{array}
$$

Collagen fraction from 2nd fin whale.

\section{U-2294. Fin whale $22 \mathrm{w}$}

$$
\begin{array}{r}
-\mathbf{3 4 0} \pm \mathbf{5 5} \\
\delta^{13} C=-22.8 \%
\end{array}
$$

Extract obtained by water treatment before acetone treatment to yield extract for U-4012. Another piece of whale used for U-2466.

\section{U-4012. Fin whale $22 \mathrm{f}$}

$$
\begin{array}{r}
-655 \pm 100 \\
\delta^{13} C=-25.1 \% 0
\end{array}
$$

Extract obtained by acetone treatment. Acetone carefully removed.

\section{U-2421. Sperm whale $1 \mathrm{c}$}

Collagen fraction.

$$
\begin{aligned}
& -35 \pm 45 \\
\delta^{1.3} C & =-13.8 \%
\end{aligned}
$$

\section{U-860. Sperm whale $1 \mathrm{f}$}

$$
\begin{array}{r}
\mathbf{5 8 0} \pm \mathbf{9 0} \\
\delta^{13} \mathrm{C}=-17.8 \% \text {. }
\end{array}
$$

Extract obtained by acetone treatment. Acetone carefully removed.

\section{U-2296. Sperm whale $2 \mathrm{c}$}

Collagen fraction from 2nd sperm whale.

$$
\begin{array}{r}
-275 \pm 60 \\
\delta^{13} C=-13.4 \%
\end{array}
$$

$$
-\mathbf{5 2 0} \pm 90
$$$$
\delta^{1.3} \mathrm{C}=-27.2 \%
$$

Extract obtained by acetone treatment. Acetone carefully removed. General Comment: samples subm on request to prove that sea mammals reflect activity of atmosphere. Because of age of whales, fat was supposed to have higher activity than collagen, which was indicated. One sample was selected to prove importance of careful removal of organic solvents when used in pretreatment of samples for radiocarbon dating. 


\section{REFERENCES}

Barker, Harold, Burleigh, Richard, and Meeks, Nigel, 1971, British Museum natural radiocarbon measurements VII: Radiocarbon, v 13, p 157-188.

Berger, Rainer, 1970, Ancient Egyptian radiocarbon chronology: Royal Soc [London] Philos Trans, ser A, v 269, p 23-36.

Birkenmajer, Krzysztof, 1960, Raised marine features of the Hornsund area, Vestspitsbergen: Studia Geol Polon, v 5, 95 p.

Birkenmajer, Krzyszt of and Olsson, I U, 1971, Radiocarbon dating of raised marine terraces at Hornsund, Spitsbergen, and problem of land uplift: Norsk Polarinst Arb, 1969, p 17-43.

Blake, W, 1975, Radiocarbon age determinations and Post-glacial emergence at Cape Storm, Southern Ellesmere Island, Arctic Canada: Geog Annals, v 57, ser A, p 1-71.

Donner, Joakim, Eronen, Matti, and Jungner, Högne, 1977, The dating of the Holocenc relative sea-level changes in Finnmark, North Norway: Norsk geog tidsskr, v 31, p 103-128.

El-Daoushy, M F A F and Olsson, I U, 1977, An improved proportional counter with high efficiency: Internatl Conf, The High Tatras, USSR, Proc, Oct 6-10, 1975, Bratislava, Czechoslovakia, p 85-90.

EI-I)aoushy, M F A F, Olsson, I U and Oro, F H, 1978, The EDTA and HCl methods of pre-treating bones: Geol Fören Stockholm Förh, no. 2.

Feyling-Hanssen, R W, 1955, Stratigraphy of the marine late-Pleistocene of Billef jorden, Vestspitsbergen: Norsk Polarinst Skr, v 107, 186 p.

Feyling-Hanssen, R W and Olsson, Ingrid, 1959-1960, Five radiocarbon datings of post glacial shorelines in central Spitsbergen: Norsk geog tidsskr, v 17, p 122-131.

Fishman, Bernard, Forbes, Hamish, and Lawn, Barbara, 1977, University of Pennsylvania radiocarbon dates XIX: Radiocarbon, v 19, p 188-228.

Follestad, B A, 1977, Toten Beskrivelse til kvart aergeologisk kart 1916 III-M 1:50 000 (Med fargetrykt kart): Norges Geol Unders, 335, p 1-45.

Follestad, B and Olsson, I U, 1979: 'The $\mathrm{C}^{14}$ age of the "Toten" mammoth, Oppland. Eastern Norway: Boreas, in press.

Hakansson, Sören, 1976, University of Lund radiocarbon dates IX: Radiocarbon, v 18, p $290-320$.

Haynes, C V, 1967, Bone organic matter and radiocarbon dating: Radioactive dating and methods of low-level counting, IAEA, Vienna, 163-168.

1968, Radiocarbon: Analysis of inorganic carbon of fossil bone and cnamel: Science, v 161, p 687-688.

Heintz, Anatol, 1956, Et nytt funn av mammutstфttann fra Norge (summary): Norsk geol tidsskr, v 36, p $17-23$.

1965 A new mammoth-find from Norway and a determination of the age of the tusk from Toten by means of $C^{14}$ : Norsk geol tidsskr, v 45, p 227-230.

Malm, A W, 1871, Hvaldjur i Sveriges Muséér år 1869: Kungl Sv Vetenskapsak Handl, V 9, no. 2.

Nordlander, Johan, 1934, Drag ur livet i Angermanland på 1500- och 1600-talen: Norrländska samlingar, no. 14 (III:4).

Olsson, Ingrid, 1958, A $\mathrm{C}^{14}$ dating station using the $\mathrm{CO}_{2}$ proportional counting method: Arkiv f Fysik, v 13, p 37-60.

1960, Uppsala natural radiocarbon measurements II: Am Jour Sci Radiocarbon Supp, v 2, p 112-128.

1966, Computer calculations of $\mathrm{C}^{14}$ determinations: Uppsala Univ Inst Physics Rept, UUIP-477, 11 p.

Olsson, Ingrid, Cazencuve, Horacio, Gustavsson, John, and Karlén, Ingvar, 1961, Uppsala natual radiocarbon measurements III: Radiocarbon, v 3, p 81-85.

Olsson, I U and El-1)aoushy, M F A F, 1978, Radiocarbon variations determined on some Egyptian samples from Dra Abu El-Naga: 9th internatl radiocarbon conf Proc, California, June 20-26, 1976 (in press).

Olsson, I U, El-Daoushy, M F A F, Abd-El-Mageed, Abdalla, and Klasson, Martin, 1974, A comparison of different methods for pretreatment of bones. I. Geol Fören Stockholm Förh, v 96, p 171-181. 
Olsson, I U, El-Gammal, S, and Göksu, Yeter, 1969, Uppsala natural radiocarbon measurements IX: Radiocarbon, v 11, p $515-544$.

Olsson, I U, and Kilicci, Serap, 1964, Uppsala natural radiocarbon measurements IV: Radiocarbon, v 6, p 291-307.

Olsson, I U, Klasson, Martin, and Abd-El-Mageed, Abdalla, 1972, Uppsala natural radiocarbon measurements XI: Radiocarbon, v 14, p 247-271.

Olsson, I U and Piyanuj, Piya, 1965, Uppsala natural radiocarbon measurements V: Radiocarbon, v 7, p 315-330.

Olsson, I U, Stenberg, Allan, and Göksu, Yeter, 1967, Uppsala natural radiocarbon measurements VII: Radiocarbon, v 9, p 454-470. 